\title{
13. SOME ROCKET-OBSERVATIONAL EVIDENCES FOR INVISIBLE FLARES
}

\section{N. P. BARABASHEV AND I. M. GORDON}

Bright chromospheric flares are sometimes not accompanied by the outbursts of radioemission of the Sun, S.I.D.'s and increases of intensity of cosmic rays; at the same time some faint flares can be followed sometimes by these remarkable events [r]. It is natural to consider all these events, including the chromospheric emission, as different manifestations of one process in which the creation of relativistic electrons plays the chief part [2].

From this point of view, we can expect that this process can proceed in certain cases in such a way that there is no emission in the chromosphere in the region where the other events take place. These events may be called 'invisible flares'. Recently we examined the solar observational data for the first nine months of 1956 [3]. Twenty new 'invisible flares' were discovered.

Direct observations have been made of the intensity of $\mathrm{L} \alpha$ and $\mathrm{X}$-ray radiation from the Sun during these flares [4].

Our point of view [2] as to the cause of the ionospheric disturbances (X-rays instead of $\mathrm{L} \alpha$ ) is confirmed.

A suggestion that this radiation arises in the regions of the corona overheated at the time of flares [4] faces serious difficulties [5]. The fade-outs usually begin simultaneously with the appearance of the chromospheric flares and sometimes before them. Further, because of the low density of the corona the time of relaxation in it is of the order of an hour. Therefore, if the heating of the corona was the source of the X-ray radiation there would be a considerable retardation of fade-outs. The lack of retardation rules out this explanation, and provides some evidence in favour of the suggestion that the X-ray radiation is a synchrotron radiation of relativistic electrons which arise at the time of a flare [2].

\section{RE FE RE N CES}

[1] Barabashev, N. P. and Gordon, I. M. Bull. 'Solar Data', Acad.Sci., U.R.S.S. 1o, 46, 1954; C.R. Acad. Sci. Ukr. R.S.S., no. I, 1954.

[2] Gordon, I. M. Ibid. 10, 49, I954; C.R. Acad. Sci. Ukr. R.S.S. 94, 8I3, 1954.

[3] Quarterly Bulletin Solar Activity, nos. II3, I14, II5.

[4] Chubb, T. A. et al. Nature, Lond. 179, 861, I957.

[5] Gordon, I. M. Trans. Kharkov Obs. 12, 15, 1957.

\section{FLARE ENERGY FROM MAGNETIC FIELDS}

\section{T. GOLD AND F. HOYLE}

It is important to realize that, although the dissipation of energy in flares takes place at the expense of the magnetic field, there can be no appreciable destruction of the magnetic component generated by electric currents below the photosphere. Only the component generated by currents in the chromosphere and corona can be thus destroyed.

The question therefore arises as to how electric currents can be generated in the solar atmosphere. Atmospheric mass motions are a possible source of such currents, but it is easy to see that this particular source is neither sufficiently energetic nor sufficiently localized to explain the dissipation that takes place in flares.

We consider the main source of atmospheric electric currents to be motions that lie at the photosphere. In particular the lines of force of a filament can be twisted by rotary motions taking place at the photospheric roots of the filament. This process carries energy, via the magnetic field, into the atmosphere. With continued twisting such a filament elongatesitself and becomes thinner as it stretches (a process probably observed in prominence arches). 